Bundesgesundheitsbl 2022 $65: 209-219$ https://doi.org/10.1007/s00103-021-03480-0 Eingegangen: 2. August 2021

Angenommen: 10. Dezember 2021

Online publiziert: 13. Januar 2022

(c) Der/die Autor(en) 2022

\author{
Johanna Mitterreiter $\cdot$ Heinrich Scheiblauer ${ }^{2}$ Sarah Fiedler ${ }^{3}$ Julia Kreß ${ }^{1}$ \\ 'Fachgebiet Molekulare Virologie, Paul-Ehrlich-Institut, Langen, Deutschland \\ ${ }^{2}$ Prüflabor für In-vitro-Diagnostika, Paul-Ehrlich-Institut, Langen, Deutschland \\ ${ }^{3}$ Abteilung Sicherheit von Arzneimitteln und Medizinprodukten, Paul-Ehrlich-Institut, Langen, \\ Deutschland
}

\title{
Sicherheit von Blut und Blutprodukten: Testmethoden zum Nachweis der Hepatitisviren B, C und E
}

Testung auf HAV oder HDV. Während HAV hauptsächlich über die fäkalorale Route übertragen wird, ist HDV helfervirusabhängig und benötigt das Oberflächenprotein von HBV (HBsAg) als Hüllprotein, wodurch es zusammen mit dem Screening auf HBsAg abgedeckt wird.

Dieser Artikel beschreibt die aktuell in Deutschland geltenden Regularien zur Sicherheit von Blut und Blutprodukten im Hinblick auf transfusionsbedingte HBV-, HCV- und HEV-Übertragungen sowie die dafür eingesetzten Screeningmethoden. Zudem wird der Erfolg dieser Maßnahmen und somit die Sicherheit von in Deutschland verwendeten Blutprodukten bewertet.

\section{Regularien in Deutschland}

Europaweit regeln die Richtlinie 2002/ 98/EG [5] und die nachfolgenden technischen Richtlinien des Europäischen Parlaments und des Rates die grundlegenden Anforderungen für Qualitätsund Sicherheitsstandards von menschlichem Blut und Blutbestandteilen für die Transfusionsmedizin. In Deutschland erfolgt die Umsetzung dieser Direktiven durch das Transfusionsgesetz (TFG; [6]), das Arzneimittelgesetz (AMG; [7]) und nachfolgende Verordnungen. Detaillierte Anforderungen an das Blutspendewesen in Deutschland sind in der Hämotherapie-Richtlinie [8] der Bundesärztekammer festgelegt. Der Arbeitskreis Blut, ein Expertengremium angesiedelt am Robert Koch-Institut, berät zudem die Bundes- und Landesbehörden in Sachen Blutsicherheit und veröffentlicht seine Beschlüsse als sogenannte Voten. Darüber hinaus kann das Paul-Ehrlich-Institut (PEI) als zuständige Bundesoberbehörde bei neuen wissenschaftlichen Erkenntnissen in einem Stufenplanverfahren spezielle Maßnahmen zur Erhöhung der Sicherheit von Blutprodukten vorschreiben.

In einem derartigen Stufenplanverfahren führt das PEI je nach Wissensstand zunächst einen Informationsaustausch mit den betroffenen Blutspendeeinrichtungen durch, um den Grad der Gefährdung zu erfassen und mögliche Maßnahmen der Risikominimierung zu diskutieren. Das PEI kann auch direkt $\mathrm{zu}$ einer beabsichtigten Auflage anhören und so Informationen zur Machbarkeit erhalten, die dann im finalen Stufenplanbescheid berücksichtigt werden können. Bei Dringlichkeit kann das PEI auch ohne Anhörung gemäß $\$ 28$ AMG die Zulassung mit Auflagen verbinden, um die Sicherheit aufrechtzuerhalten. Bisher veröffentlichte Stufenplanbescheide des PEI betrafen Auflagen zum Spenderscreening, also zur Testung auf AntigenAntikörper- bzw. Erregerbestandteile, Auflagen zur Spenderauswahl oder auch Auflagen, die sich auf Herstellungsschritte bezogen.

Die aktuell gültigen Anforderungen an das Screening von Blutspenden auf 
Tab. 1 Umfang des Blutspenderscree-

\begin{tabular}{|c|c|c|}
\hline Marker & Serologie & NAT \\
\hline HBV & Anti-HBC ${ }^{a}, \mathrm{HBsAg}$ & - \\
\hline $\mathrm{HCV}$ & Anti-HCV & HCV \\
\hline HEV & - & HEV \\
\hline HIV-1/2 & Anti-HIV-1/2 & HIV-1/2 \\
\hline WNV ${ }^{b}$ & - & WNV \\
\hline Syphilis & $\begin{array}{l}\text { Anti-Treponema } \\
\text { pallidum }\end{array}$ & - \\
\hline \multicolumn{3}{|c|}{$\begin{array}{l}{ }^{a} \text { Anti-HBc-reaktive und HBsAg-negative Blut- } \\
\text { spenden dürfen freigegeben werden, wenn } \\
2 \text { weitere, vom initialen verwendeten Scree- } \\
\text { ningtest verschiedene Anti-HBc-Testsysteme } \\
\text { (oder ein Anti-HBc-Bestätigungstest) und eine } \\
\text { Testung auf HBV-Genome mittels NAT (Mindest- } \\
\text { sensitivität } \leq 5 \text { IE/mL) negative Testergebnisse } \\
\text { ergeben }[10,38] \\
{ }^{b} \text { WNVWest-Nil-Virus. Von der saisonalen } \\
\text { (1.06.-30.11.), 4-wöchigen Spenderrückstellung } \\
\text { kann durch ein WNV-NAT-Screening abgewi- } \\
\text { chen werden } \\
\text { NAT Nukleinsäureamplifikationstechniken, } \\
\text { HBV Hepatitis-B-Virus, HCV Hepatitis-C-Virus, } \\
\text { HEV Hepatitis-E-Virus, HIV Humanes Immunde- } \\
\text { fizienz-Virus, WNVWest-Nil-Virus, HBC Hepatitis- } \\
\text { B-Virus Oberflächenprotein, HBsAg Hepatitis-B- } \\
\text { Virus Core-Antigen }\end{array}$} \\
\hline
\end{tabular}

Virusinfektionen sind in • Tab. 1 zusammengefasst. Allgemein wird zwischen serologischen Screeningmethoden auf Antigene bzw. Antikörper und Nukleinsäureamplifikationstechniken (NAT) zur Testung auf Virusgenom unterschieden. Während für $\mathrm{HCV}$ sowohl die Testung der Antikörper als auch eine HCV-NAT vorgeschrieben sind, ist für das HBV-Screening eine serologische Testung auf Anti-HBc-Antikörper und das HBs-Antigen ausreichend [9-11]. In den meisten Blutspendeeinrichtungen wird außerdem eine NAT-Testung auf HBV-DNA routinemäßig auf freiwilliger Basis durchgeführt. Für HEV wurde $a b$ 2020 die HEV-NAT verpflichtend für das Screening von Blutspenden eingeführt [12].

Blutspendeeinrichtungen und pharmazeutische Unternehmer müssen alle für das Blutspenderscreening eingesetzten Prüfverfahren für die in • Tab. 1 gelisteten Infektionsmarker beim PEI anzeigen. Diese werden dann auf ihre Eignung überprüft und bei Erfüllung aller Anforderungen in eine Datenbank „Spendertestung" aufgenommen [11]. - Tab. 2 enthält eine Übersicht der 2021 in Deutsch- land gemeldeten Screeningtests für HBV, HCV und HEV. Neben kommerziell erhältlichen Tests, die durch ein Zertifizierungsverfahren (CE) speziell für das Blutspenderscreening zugelassen sind, dürfen auch solche kommerziellen Tests verwendet werden, die nicht primär für das Blutscreening zugelassen sind (sog. Off Label Use). Auch eigens etablierte „InHaus“-Methoden dürfen verwendet werden - sofern sie den vom PEI festgelegten Anforderungen entsprechen und die notwendigen Validierungen vorgenommen wurden.

\section{Prävalenz, Übertragung, Infektionsverläufe und Testung bei HBV, HCV und HEV}

Taxonomisch betrachtet handelt es sich bei Hepatitisviren um sehr unterschiedliche Viren, die ihren Namen nur aufgrund der gleichen primär induzierten Erkrankung teilen.

\section{HBV}

$\mathrm{HBV}$ ist das am weitesten verbreitete $\mathrm{He}$ patitisvirus mit weltweit etwa 250 Mio. chronisch Infizierten und geschätzten 887.000 Todesfällen im Jahr 2015 [1]. In Deutschland wurden 2019 knapp 9000 HBV-Fälle gemeldet. Die Übertragung erfolgt durch Kontakt mit Blut oder anderen Körperflüssigkeiten [13]. Der primäre Übertragungsweg ist in Deutschland der sexuelle Kontakt. Im Gegensatz zu HCV und HEV gibt es eine effektive Impfung gegen HBV. Diese ist seit 1995 Bestandteil der empfohlenen Standardimpfungen für Säuglinge, Kinder, Jugendliche und Erwachsene. HBV gehört zur Familie der Hepadnaviridae und hat ein zirkuläres, teilweise doppelsträngiges DNA-Genom. Dieses wird von einem Kapsid aus Core-Antigen (HBcAg) umschlossen, welches wiederum von einer Hülle umgeben ist, die aus dem Oberflächenprotein HBsAg (engl. „surface antigen“) aufgebaut ist [14].

Eine Infektion mit HBV kann sehr unterschiedlich verlaufen. Während sie bei etwa einem Drittel der Erwachsenen asymptomatisch verläuft, kommt es bei bis zu $10 \%$ der Patienten mit akuter Hepatitis zu einer Progression zum chro- nischen Verlauf. Im Laufe einer chronischen HBV-Infektion besteht die Gefahr der Entwicklung einer Leberzirrhose oder eines Leberzellkarzinoms. Die Infektions- bzw. Krankheitsstadien können anhand der Kombination von HBV-Genom, HBV-Antigenen und Antikörpern gegen HBV-Proteine nachgewiesen werden. Die Inkubationszeit von der HBVInfektion bis zur Etablierung von klinischen Symptomen beträgt durchschnittlich 60 bis 120 Tage. Für die Transfusionsmedizin ist jedoch bereits die Phase vor Einsetzen der klinischen Symptome ausschlaggebend, wenn sich das Virus asymptomatisch vermehrt.

Eine HBV-Infektion lässt sich meist erst nach einigen Wochen durch den Nachweis von HBV-DNA detektieren, gefolgt vom HBsAg-Nachweis weitere Wochen später, bevor schließlich noch weitere Wochen später auch Antikörper gegen $\mathrm{HBc}(\mathrm{Anti}-\mathrm{HBc})$ gebildet und detektiert werden können (• Abb. 1). Bei asymptomatischer Infektion und einer ausheilenden akuten Hepatitis nimmt die Menge an HBV-DNA und HBsAg während des Infektionsverlaufes wieder $a b$, während bei einer chronischen Infektion HBV-DNA und HBsAg oft über Monate und Jahre nachweisbar sind. Anti-HBcAntikörper sind im Allgemeinen jahrebis lebenslang im Körper nachweisbar $[14,15]$.

Aktuell ist für Spenderblut in Deutschland die Testung auf HBsAg und Anti$\mathrm{HBc}$ vorgeschrieben (siehe oben). Um die sogenannte diagnostische Fensterperiode - die Zeit, in der das Virus im Blut vorhanden ist, aber noch nicht nachgewiesen werden kann, - zu verringern, testen die meisten Blutspendeeinrichtungen zusätzlich freiwillig auf HBV-DNA. Die diagnostische Fensterperiode kann somit um durchschnittlich 2,1-4,9 Tage im Vergleich zur HBsAg-Testung reduziert werden (siehe Abschnitt zur HBsAgTestung).

HCV

$\mathrm{HCV}$ ist ebenfalls weltweit verbreitet mit geschätzten 71 Mio. chronisch Infizierten und 399.000 Todesfällen im Jahr 2015 [1]. Die Anzahl der 2019 in Deutschland gemeldeten HCV-Erstdia- 
Bundesgesundheitsbl 2022 -65:209-219 https://doi.org/10.1007/s00103-021-03480-0

(c) Der/die Autor(en) 2022

\section{J. Mitterreiter · H. Scheiblauer · S. Fiedler ·J. Kreß}

\section{Sicherheit von Blut und Blutprodukten: Testmethoden zum Nachweis der Hepatitisviren B, C und E}

\section{Zusammenfassung}

Infektionen mit den Hepatitisviren $B, C$ und $E$ (HBV, HCV, HEV) sind über das Blut übertragbar und können schwere akute und chronische Leberentzündungen hervorrufen. Um die Sicherheit von Spenderblut zu gewährleisten und Empfänger vor Virusübertragungen zu schützen, werden Blutkonserven in Deutschland mit Nukleinsäureamplifikationstechniken (NAT) auf Virusgenom sowie mit serologischen Methoden auf virale Antigene und humane Antikörper getestet. In diesem Beitrag werden die entsprechenden Regularien zur Sicherheit von Blut und Blutprodukten in Deutschland sowie die verschiedenen Screeningmethoden beschrieben. Die Sicherheit der Blutprodukte wird bewertet.
Beim Spenderscreening angewandte NATMethoden basieren auf Technologien der Polymerasekettenreaktion (PCR) oder auf isothermen Verfahren, wie der Transcriptionmediated Amplification (TMA), welche einen hochempfindlichen Nachweis einer Virusinfektion in Spenderblut ermöglichen und so zu einer Verkürzung der diagnostischen Fensterperiode beitragen. Bereits seit den 1970er-Jahren wird zur Erkennung einer möglichen HBV-Infektion ein Screening des viralen Oberflächenproteins ( $\mathrm{HBsAg}$ ) gefordert. Die verpflichtende Einführung der Testung auf HCV-spezifische Antikörper 1992, der HCV-NAT-Testung 1999 und des Anti-HBc-Spenderscreenings 2006 sowie die nichtverpflichtende, von den meisten Blutspendeeinrichtungen freiwillig durchgeführte HBV-NAT-Testung haben die Sicherheit von Blutprodukten deutlich verbessert und Übertragungsfälle auf seltene Einzelfälle in der frühen diagnostischen Fensterperiode reduziert. Der Erfolg der 2020 in Deutschland eingeführten HEVNAT-Testung von Spenderblut wird sich in den kommenden Jahren bemessen lassen. Neben der Spendertestung ergänzen Maßnahmen zur Spenderauswahl und Pathogeninaktivierung das Sicherheitssystem für Blutspenden in Deutschland.

\section{Schlüsselwörter}

Hepatitisviren - Blutspenderscreening - NAT . Antigentest · Blutsicherheit

\section{Safety of blood and blood products: test methods for the detection of hepatitis B, C, and E virus}

\section{Abstract}

Infections with hepatitis $B, C$, and $E$ virus (HBV, HCV, and HEV) can be transmitted via blood and cause severe acute or chronic liver infections. To ensure the safety of blood donations and protect recipients from virus transmissions, blood donations in Germany are tested for viral genomes using nucleic acid amplification techniques (NATs) as well as for viral antigens and antibodies by serological testing. This article describes the relevant regulations on the safety of blood and blood products in Germany and the various screening methods. The safety of blood products is assessed.

Currently used NAT methods for detection of hepatitis viruses are based either on polymerase chain reaction (PCR) or isothermal methods such as transcription-mediated amplification (TMA), which enable a highly sensitive detection of viral infections and thereby contribute to the reduction of the diagnostic window. Antigen tests for the detection of viral surface protein of hepatitis $B$ virus in blood donations were introduced in the 1970s in order to prevent potential transmissions. Since the introduction of mandatory testing for HCV-specific antibodies in 1992, HCV NAT testing in 1999, anti-HBC antibody testing in 2006, and the non-mandatory HBV NAT, which is voluntarily performed by most of the blood establishments, blood safety has increased tremendously. Only a few isolated cases of transfusion-transmitted infections in the early window period have been reported since. The success of the recent introduction of mandatory HEV NAT testing in 2020 will have to be assessed in the upcoming years. Besides blood donor screening, the system for blood safety in Germany is supplemented by additional measures for donor selection and pathogen inactivation.

\section{Keywords}

Hepatitis virus - Blood screening - NAT . Antigen testing $\cdot$ Blood safety gnosen betrug 5940 Fälle. Das Virus wird fast ausschließlich durch den Kontakt mit kontaminiertem Blut übertragen. Während in Deutschland der unsichere injizierende Drogenkonsum den häufigsten Übertragungsweg darstellt, sind weltweit zusätzlich nosokomiale Übertragungen für einen großen Teil der Infektionen verantwortlich, die in Deutschland keine Rolle spielen [13]. HCV ist ein Hepacivirus aus der Familie der Flaviviridae mit einem einzelsträngigen RNA-Genom positiver Polarität, welches von einem Kapsid und einer Virushülle umgeben ist [16].

Etwa $75 \%$ der HCV-Infizierten bleiben symptomfrei, der Rest entwickelt nach einer Inkubationszeit von ca. 40 bis 120 Tagen eine akute, aber meist recht milde Hepatitis. Die Mehrzahl der akuten Hepatitiden persistiert allerdings in Form einer chronischen Hepatitis jahrelang im Körper. Als Spätfolgen entwickeln etwa 16-20\% der Patienten mit chronischer Hepatitis $\mathrm{C}$ nach 20 Jahren eine Leberzirrhose, was wiederum mit einem erhöhten Risiko für das
Leberzellkarzinom einhergeht. HCVInfektionen werden beim Spenderscreening über den Nachweis von HCV-RNA und Anti-HCV-Antikörpern detektiert. Eine Serokonversion findet 45-51 Tage nach HCV-Infektion statt [17], gebildete Antikörper bleiben daraufhin jedoch unabhängig vom klinischen Krankheitsbild meist lebenslang nachweisbar. HCVRNA ist im Gegensatz dazu bereits deutlich früher nachweisbar, nimmt jedoch im Laufe einer ausheilenden akuten HCV-Hepatitis wieder ab (• Abb. 1). Bei chronischen Verläufen ist das HCV- 
Tab. 2 Übersicht der 2021 in Deutschland gemeldeten CE-Screeningtests für Blutspenden

\begin{tabular}{|c|c|c|c|c|}
\hline Marker & Testname & Hersteller & Testprinzip & Messprinzip \\
\hline HBV DNA & cobas MPX (6800/8800 System) & Roche Diagnostics & Screening (multiplex) & PCR \\
\hline HBV DNA & cobas TaqScreen MPX Test, v2.0 & Roche Diagnostics & Screening (multiplex) & PCR \\
\hline HBV DNA & Procleix Ultrio Elite Assay & Grifols Diagnostic Solutions Inc & Screening (multiplex) & TMA \\
\hline HBV DNA & Procleix Ultrio Plus Assay & Grifols Diagnostic Solutions Inc & Screening (multiplex) & TMA \\
\hline HBV DNA & Procleix Ultrio Assay & Grifols Diagnostic Solutions Inc & Screening (multiplex) & TMA \\
\hline HBV DNA & Virus Screening PCR Kit, v1.3 & GFE Blut mbH & Screening (multiplex) & PCR \\
\hline HCV RNA & cobas MPX (6800/8800 System) & Roche Diagnostics & Screening (multiplex) & PCR \\
\hline HCV RNA & cobas TaqScreen MPX Test, v2.0 & Roche Diagnostics & Screening (multiplex) & PCR \\
\hline HCV RNA & Procleix Ultrio Elite Assay & Grifols Diagnostic Solutions Inc & Screening (multiplex) & TMA \\
\hline HCV RNA & Procleix Ultrio Plus Assay & Grifols Diagnostic Solutions Inc & Screening (multiplex) & TMA \\
\hline HCV RNA & Procleix Ultrio Assay & Grifols Diagnostic Solutions Inc & Screening (multiplex) & TMA \\
\hline HCV RNA & Virus Screening PCR Kit, v1.3 & GFE Blut mbH & Screening (multiplex) & PCR \\
\hline HEV RNA & cobas HEV (6800/8800 System) & Roche Diagnostics & Screening & PCR \\
\hline HEV RNA & Procleix HEV Assay & Grifols Diagnostic Solutions Inc & Screening & TMA \\
\hline Anti-HBc & Alinity i Anti-HBc II Reagenz & Abbott GmbH & Indirekt (Anti-Human-IgG, -IgM) & CMIA \\
\hline Anti-HBc & Alinity s Anti-HBc Reagenz & Abbott GmbH & Indirekt (Anti-Human-IgG, -IgM) & CMIA \\
\hline Anti-HBc & Architect Anti-HBc II Reagenz & Abbott GmbH & Indirekt (Anti-Human-IgG, -IgM) & CMIA \\
\hline Anti-HBC & Prism HBcore Reagenz & Abbott GmbH & Kompetitiv & CLIA \\
\hline Anti-HBC & Access $\mathrm{HBc} \mathrm{Ab}$ & Beckman Coulter & Indirekt (Protein A) & CLIA \\
\hline Anti-HBC & Monolisa Anti-HBc Plus & Bio-Rad & Indirekt (Anti-Human-IgG, -IgM) & ELISA \\
\hline Anti-HBc & Liaison Anti-HBC & DiaSorin S.p.A. & Kompetitiv & CLIA \\
\hline Anti-HBc & Murex anti-HBc (total) & DiaSorin S.p.A. - UK Branch & Kompetitiv & ELISA \\
\hline Anti-HBC & Anti-HBC & Roche Diagnostics & Kompetitiv & ECLIA \\
\hline Anti-HBC & Elecsys Anti-HBc II & Roche Diagnostics & Kompetitiv & ECLIA \\
\hline Anti-HBC & Advia Centaur Anti-HBc Total (HBcT) & Siemens Healthcare Diagnostics & Sandwich & CLIA \\
\hline Anti-HBc & Advia Centaur HBc Total 2 (HBcT2) & Siemens Healthcare Diagnostics & Sandwich & CLIA \\
\hline Anti-HBC & Atellica IM Anti Hepatitis B core Total (HBcT) & Siemens Healthcare Diagnostics & Sandwich & CLIA \\
\hline Anti-HBC & Atellica IM HBc Total 2 (HBcT2) & Siemens Healthcare Diagnostics & Sandwich & CLIA \\
\hline $\mathrm{HBsAg}$ & Alinity i HBsAg Qualitative II Reagenz & Abbott GmbH & Sandwich & CMIA \\
\hline $\mathrm{HBsAg}$ & Alinity i HBsAg Reagenz & Abbott GmbH & Sandwich (quantitativ) & CMIA \\
\hline $\mathrm{HBsAg}$ & Alinity s HBsAg Reagenz & Abbott GmbH & Sandwich & CMIA \\
\hline $\mathrm{HBsAg}$ & Architect HBsAg Qualitative II Reagenz & Abbott GmbH & Sandwich & CMIA \\
\hline $\mathrm{HBsAg}$ & Prism HBsAg Reagenz & Abbott GmbH & Sandwich & CLIA \\
\hline $\mathrm{HBsAg}$ & Access HBsAg & Beckman Coulter & Sandwich & CLIA \\
\hline $\mathrm{HBsAg}$ & Liaison XL murex HBsAg Quant & DiaSorin S.p.A. & Sandwich (quantitativ) & CLIA \\
\hline $\mathrm{HBsAg}$ & Elecsys HBsAg II & Roche Diagnostics & Sandwich & ECLIA \\
\hline $\mathrm{HBsAg}$ & HBsAg II & Roche Diagnostics & Sandwich & ECLIA \\
\hline $\mathrm{HBsAg}$ & Advia Centaur HBsAg II (HBsIl) & Siemens Healthcare Diagnostics & Sandwich & CLIA \\
\hline $\mathrm{HBsAg}$ & Atellica IM Hepatitis B surface Antigen II (HBsll) & Siemens Healthcare Diagnostics & Sandwich & CLIA \\
\hline
\end{tabular}

Genom meist durchgehend nachweisbar $[15,16]$.

\section{HEV}

HEV ist ein kleines, unbehülltes Virus der Familie der Hepeviridae mit einem einzelsträngigen, positiv-polarisierten RNA-Genom. HEV wurde ursprünglich eher als ein Problem in Entwicklungsländern betrachtet, das hauptsächlich als reiseassoziierte Krankheit in Industrienationen eingeschleppt wird. In den letzten 10 Jahren hat HEV jedoch weltweit an Bedeutung gewonnen mit geschätzten 20Mio. HEV-Infektionen jährlich [1]. Hepatitis E und ihr Erreger HEV weisen je nach Region und Genotyp deutliche Unterschiede in Epidemiologie und Klinik auf. In vielen Ländern Asiens und Afrikas kommen hauptsächlich die HEV-Genotypen 1 und
2 endemisch vor. Übertragung erfolgt hier aufgrund unzureichender Trinkwasser- und Lebensmittelhygiene meist durch mit menschlichen Fäkalien kontaminiertes Trinkwasser. Für die HEVGenotypen 1 und 2 ist der Mensch das einzige bekannte Reservoir.

In Deutschland sowie anderen Industrienationen Europas und Nordamerikas ist vor allem der HEV-Genotyp 3 (HEV3) endemisch verbreitet. Die Übertra- 


\begin{tabular}{|c|c|c|c|c|}
\hline Marker & Testname & Hersteller & Testprinzip & Messprinzip \\
\hline Anti-HCV & Alinity i Anti-HCV Reagenz & Abbott GmbH & Indirekt (Anti-Human-lgG/lgM) & CMIA \\
\hline Anti-HCV & Alinity s Anti-HCV Reagenz & Abbott GmbH & Sandwich & CMIA \\
\hline Anti-HCV & Architect Anti-HCV Reagenz & Abbott GmbH & Indirekt (Anti-Human-IgG/lgM) & CMIA \\
\hline Anti-HCV & Prism HCV Reagenz & Abbott GmbH & Indirekt (Anti-Human-IgG) & CLIA \\
\hline Anti-HCV & Liaison XL murex HCV Ab & DiaSorin S.p.A. & Indirekt (Anti-Human-IgG) & CLIA \\
\hline Anti-HCV & HCV 3.0 ELISA Test System with Enhanced SAVe & Ortho - Clinical Diagnostics & Indirekt (Anti-Human-lgG) & ELISA \\
\hline Anti-HCV & Anti-HCV II & Roche Diagnostics & Sandwich & ECLIA \\
\hline Anti-HCV & Elecsys Anti-HCV II & Roche Diagnostics & Sandwich & ECLIA \\
\hline Anti-HCV & Advia Centaur Anti-HCV & Siemens Healthcare Diagnostics & Indirekt (Anti-Human-IgG) & CLIA \\
\hline Anti-HCV & Atellica IM Hepatitis C (aHCV) & Siemens Healthcare Diagnostics & Indirekt (Anti-Human-IgG) & CLIA \\
\hline $\mathrm{HCV} \mathrm{Ag} / \mathrm{Ab}$ & Murex HCV Ag/Ab Combination & DiaSorin S.p.A. - UK Branch & Sandwich & ELISA \\
\hline
\end{tabular}

gung erfolgt hauptsächlich zoonotisch über den Verzehr von unzureichend gegartem Fleisch und daraus hergestellten Produkten. Das Hausschwein ist hierbei das vermutlich wichtigste tierische Reservoir. Das Virus kann auch parenteral (z.B. durch kontaminierte Blutprodukte) übertragen werden. Mensch-zuMensch-Übertragungen von Infektionen mit HEV-3 im Alltag sind jedoch extrem selten. Die Zahl der in Deutschland gemeldeten Fälle steigt seit Jahren kontinuierlich an und lag 2019 bei 3724, was aber auch auf die allgemein vermehrte Aufmerksamkeit für HEV in der Wissenschaft und Medizin zurückzuführen sein kann [2]. Eine Studie von 2014 zeigte eine Seroprävalenz von $6,8 \%$ unter deutschen Blutspendern [18]. Aufgrund des vermehrten Auftretens transfusionsbedingter HEV-Übertragungen $[4,19$, 20] wurde das Screening von HEV-RNA in Blutspenden mittlerweile in Deutschland und vielen anderen europäischen Ländern verpflichtend angeordnet (siehe oben).

Eine HEV-Infektion verläuft in der Mehrzahl der Fälle asymptomatisch, führt jedoch nach einer Inkubationszeit von ca. 2-6 Wochen in etwa 5-20\% zu einer akuten Hepatitis, welche in der Regel selbstlimitierend wieder ausheilt. Eine chronische Verlaufsform wurde bisher meist nur in immunsupprimierten Patienten diagnostiziert. HEV-RNA im Blut ist kurz vor Beginn der Symptome gegen Ende der Inkubationszeit detektierbar, kann jedoch etwa 2-4 Wochen nach Krankheitsbeginn nicht mehr nachge- wiesen werden. Serokonversion findet kurz nach akutem Krankheitsbeginn statt und führt $\mathrm{zu}$ einem meist persistierenden Anti-HEV-Antikörpertiter (• Abb. 1; [3, 21]).

\section{Nachweis erregerspezifischer Nukleinsäuren}

Erregerspezifische Nukleinsäuren sind bei den meisten blutübertragenen Viren der erste im Blut nachweisbare Infektionsmarker (siehe oben). NAT-Methoden ermöglichen es somit, Hepatitisvirusinfektionen früh $\mathrm{zu}$ erkennen und die diagnostische Fensterperiode, in der das Virus zwar bereits im Körper vorhanden, aber noch nicht nachweisbar ist, möglichst gering zu halten. Zudem lässt die Anwesenheit von Virusgenom direkt auf eine hohe Ansteckungsgefahr schließen.

Zum Nachweis von erregerspezifischen Nukleinsäuren werden beim Spenderscreening 2 verschiedene Techniken eingesetzt. Die überwiegende Anzahl (85-88\%) deutscher Blutspendeeinrichtungen verwendet die (Echtzeit-)Polymerasekettenreaktion (PCR) zum Nachweis von Hepatitisviren in Spenderblut, während die Transcription-mediated Amplification (TMA) in 12-15\% der deutschen Blutspendeeinrichtungen zum Einsatz kommt (• Abb. 2). Andere NAT-Techniken, wie z.B. LAMP (Loop-mediated Isothermal Amplification), werden aktuell nicht angewendet. Mithilfe von PCR und TMA können bereits geringe Mengen an Erregergenom vervielfäl- tigt, detektiert und somit nachgewiesen werden.

Aktuell wird für das Spenderscreening in Deutschland eine Mindestnachweisgrenze von 5000 Internationalen Einheiten (IE) HCV-RNA pro mL bzw. $2000 \mathrm{IE} / \mathrm{mL}$ HEV-RNA bezogen auf die Einzelspende gefordert [11, 12]. In Deutschland ist eine Testung mit einer Poolgröße von bis zu 96 Einzelspenden gestattet - vorausgesetzt die Sensitivität der verwendeten Tests entspricht den genannten Anforderungen und entsprechende Validierungen wurden vorgenommen. Aufgrund des rasanten, technischen Fortschritts im Bereich der In-vitro-Diagnostika (IVD) wurden in den letzten 10 Jahren mehrere hochsensitive, automatisierte Systeme in den europäischen Markt eingeführt, welche bereits geringe Mengen an Erregergenom detektieren können. Die meisten heutzutage in Deutschland verwendeten NAT-Screeningsysteme (• Tab. 2) greifen auf automatisierte Plattformen zurück, welche die Extraktion der Nukleinsäure mit der Detektion des Erregers in einem Gerät vereinen. Somit sind aktuell analytische Sensitivitätsgrenzen (95\% Limit of Detection, LoD) von bis zu 1,4 IE HBV-DNA, 3,0 IE HCV-RNA bzw. 7,9IE HEV-RNA pro mL bezogen auf die Einzelspende möglich.

Da die PCR auf der Vervielfältigung von DNA beruht, wird für den Nachweis von HCV- und HEV-Erregern das RNA-Genom in einem vorherigen Schritt durch das Enzym Reverse Transkriptase in komplementäre DNA (cDNA) umge- 


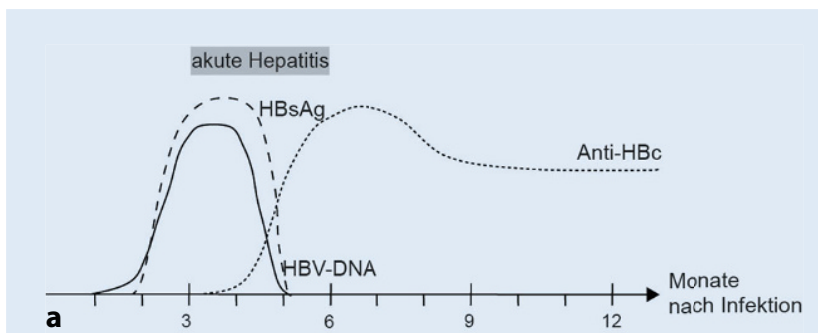

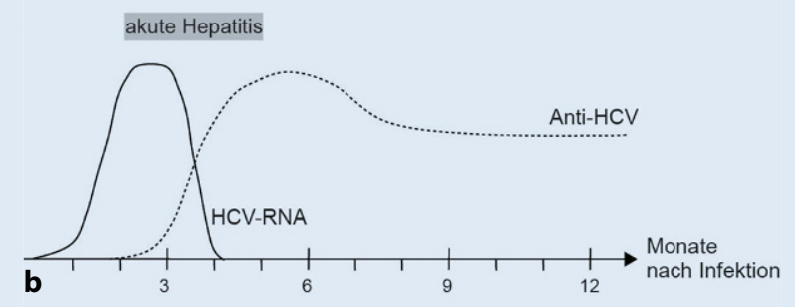

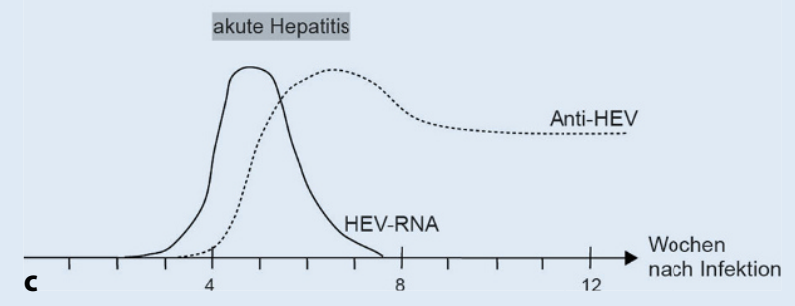

Abb. $1<$ Exemplarische Infektionsverläufe von ausheilenden Hepatitiden. Antikörper-, Nukleinsäure- und Antigenverläufe nach HBV-, HCVbzw. HEV-Infektion. a HBV, b HCV, cHEV. HBVHepatitsB-Virus, HCV Hepatitis-C-Virus, HEV Hepatitis-EVirus, $\mathrm{HBsAg} \mathrm{He}-$ patitis-B-Virus Oberflächenprotein, $H B C$ Hepatitis-BVirus Core-Antigen

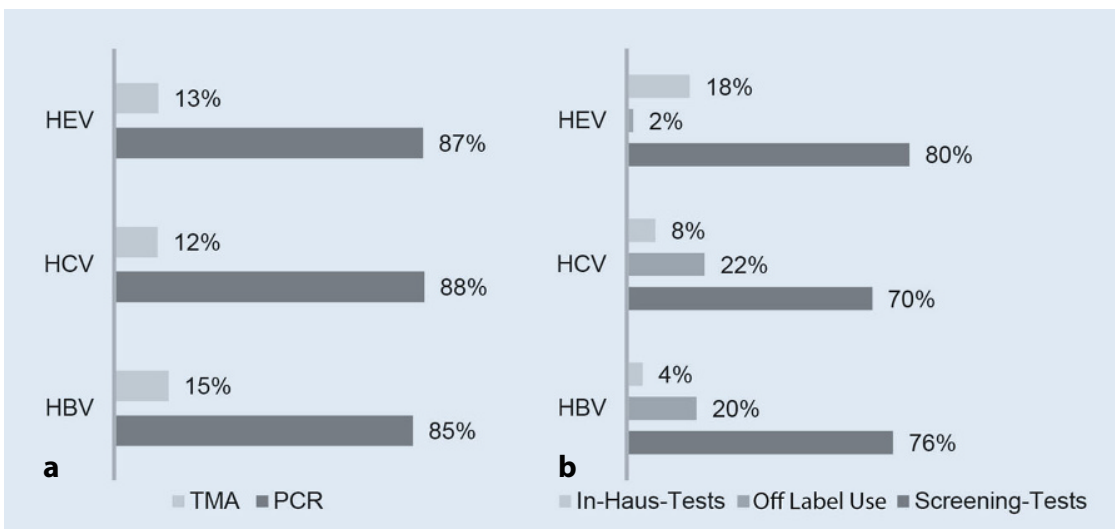

Abb. $2 \Delta$ In Deutschland angewandte NAT-Systeme zum Spenderscreening. Prozentualer Anteil in Deutschland angewandter NAT-Systeme zum Spenderscreening gemessen an der Anzahl der Blutspendeeinrichtungen und unterteilt nach verwendeter Technologie bzw. Format. TMA Transcriptionmediated Amplification, PCR Polymerase Chain Reaction. a Technologie, b Format

schrieben, bevor es zu einer zyklischen, exponentiellen Vermehrung von kurzen, gezielten Genabschnitten der Hepatitiserreger kommt. Durch ein spezifisches Primerdesign in konservierten Bereichen des Genoms ist oft die Amplifizierung eines Genomabschnittes ausreichend. Die Detektion und Quantifizierung erfolgt anhand der Echtzeit-PCR-Technologie (Real-Time-PCR) durch fluoreszenzamplifizierten Genommenge ist [22].
Kopie um, die daraufhin der RNA-Polymerase als Vorlage zur Produktion mehrerer RNA-Kopien dient, welche wiederum von der Reversen Transkriptase in DNA-Kopien umgeschrieben werden. Die entstandenen RNA-Kopien können nun mit einer erregerspezifischen, markierten Sonde detektiert werden [23].

Eine Studie des Deutschen Roten Kreuzes zeigte bereits 1999 den Nutzen und die Machbarkeit einer systematischen NAT-Testung mittels In-HausMethoden und kommerzieller PCR auf [24]. Nach der Einführung der verpflichtenden NAT-Testung ist auch der europäische Markt für kommerzielle IVD stetig gewachsen. Während vor 20 Jahren noch die Mehrzahl der Laboratorien eigens etablierte In-Haus-Methoden zum Blutspenderscreening verwendete, so greifen aktuell 70-80\% der Blutspendeeinrichtungen in Deutschland auf kommerzielle IVD zurück, die speziell für das Spenderscreening validiert und zugelassen wurden (ब Abb. 2). Die restlichen Einrichtungen verwenden entweder InHaus-Methoden oder kommerzielle IVD im Off Label Use. Sowohl bei Verwendung von IVD im Off Label Use als auch bei In-Haus-Methoden müssen die Blutspendeeinrichtungen jedoch zusätzliche Validierungsmaßnahmen vornehmen, bevor das PEI die Verwendung dieser Tests zum Spenderscreening genehmigt. Aufgrund der erst kürzlich geänderten Vorschriften zur HEV-Testung und einer geringeren Anzahl an kommerziellen HEV-NAT-Systemen auf dem europäischen Markt ist der Anteil der Einrichtungen, die HEV In-Haus-Tests durchführen, mit $18 \%$ noch deutlich höher im Vergleich zur HBV- und HCVTestung (• Abb. 2).

\section{Nachweis von Antikörpern gegen HCV}

Fluoreszenzintensität proportional zur

Die TMA ist im Gegensatz zur PCR eine isotherme Methode und vervielfältigt Genomabschnitte mithilfe der beiden Enzyme RNA-Polymerase und Reverse Transkriptase. Die Reverse Transkriptase schreibt mithilfe erregerspezifischer Primer die RNA-Zielsequenz in eine DNA-
Serologische Assays zum Nachweis von Antikörpern gegen HCV (Anti-HCV) wurden nach Entdeckung des Virus im Jahr 1989 entwickelt und seitdem kontinuierlich verbessert. Die heute verwendeten Anti-HCV-Assays der 3. Generation enthalten rekombinante Proteine oder Peptide des HCV-Kernproteins (Core) 


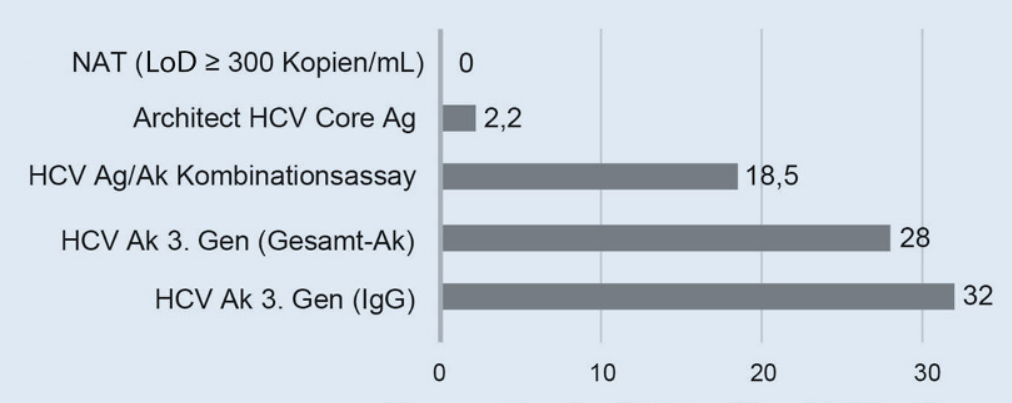

a

Tage nach erster NAT-positiver Blutabnahme

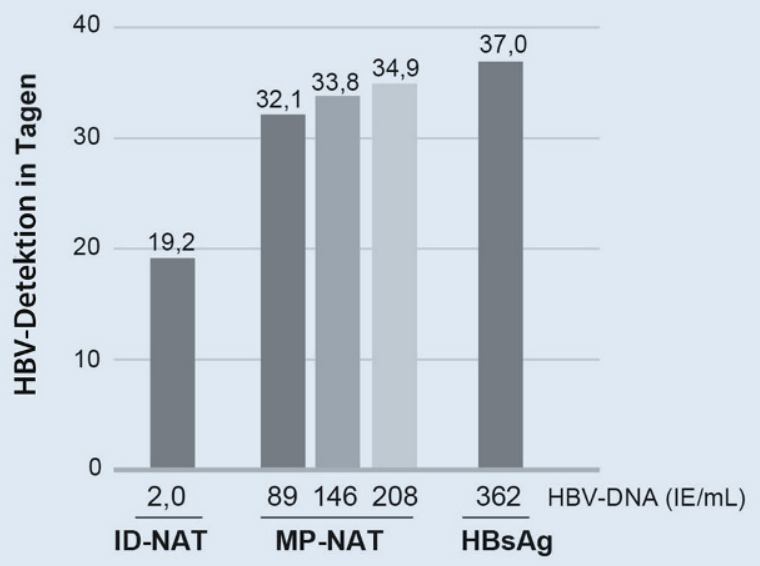

Abb. $3 \Delta$ Antikörper- und Antigennachweis von HBV und HCV nach akuter Infektion. a Zeitangaben für die diagnostische Fensterperiode nach akuter HCV-Infektion in Abhängigkeit vom verwendeten Nachweistest. b Zeitpunkt des HBV-Nachweises in 30 HBV-Serokonversionspanels. Die Nachweisgrenze (Limit of Detection) der einzelnen Methoden ist unterhalb der Säulen in internationalen Einheiten (IE) HBV-DNA/mL angegeben. MP-NAT Minipool-NAT, ID-NAT Einzelspenden-NAT. Abbildung adaptiert von Scheiblauer et al., 2020 [28]. Die Abbildungsrechte liegen bei den Autor:innen

und der nichtstrukturellen Proteine NS3, meist noch NS4 sowie in einigen Tests zusätzlich NS5. Die Sensitivitätsverbesserung gegenüber der 2. Generation ist in erster Linie auf Rekonfiguration von NS3 durch Reduktionsmittel wie Dithiothreitol (DTT) oder Cystein zurückzuführen, wodurch HCV-Verläufe, die überwiegend durch Anti-NS3 bestimmt sind, früher erkannt bzw. nicht mehr verpasst werden. Eine ausgewogene Sensitivität gegenüber den immunogenen Hauptantigenen Core und NS3 ist daher nach wie vor ein wichtiger Bestandteil bei der Bewertung von neuen Anti-HCVScreeningtests $[11,25]$. Eine weitere Verbesserung der Sensitivität wurde in den letzten Jahren durch „Sandwich“-Tests (anstelle von indirekten IgG-Tests) erreicht, die Anti-HCV-Gesamtantikörper nachweisen, wodurch HCV-Verläufe mit offenbar bisher wenig eingesetzt verglichen z.B. mit den weitverbreiteten HIV-Ag/Ak-Assays der 4. Generation. - Abb. 3a zeigt auch die diagnostische Fensterperiode für einen reinen $\mathrm{HCV}$ Antigentest, der diese im Vergleich zur PCR auf 2,2 Tage deutlich verkürzt. Dieser Test wird jedoch nicht im Blutspenderscreening eingesetzt.

Die Genotypdiversität von HCV zeigte keinen Einfluss auf die Sensitivität der Anti-HCV-Assays [26]. Offenbar scheint die Kombination der Antigenregionen von Core, NS3 und NS4, mit denen die Anti-HCV-Tests beschichtet sind, ausreichend konserviert $\mathrm{zu}$ sein, um alle 6 HCV-Genotypen zuverlässig erfassen zu können.

\section{Nachweis des Hepatitis-B- Oberflächenantigens}

Der Nachweis des Hepatitis-B-Oberflächenantigens basiert auf dem Nachweis der „a“-Determinante des HBV-S-Gens, das bei allen HBV-Isolaten vorkommt. Als Testprinzip wird in der Regel ein Sandwichtestdesign verwendet, bei dem das HBs-Antigen durch 2 verschiedene spezifische Antikörper von 2 Seiten zwischen Fest- und Konjugatphase gebunden wird. Die Sensitivität ist an einem internationalen Standard angeglichen und wird in Internationalen Einheiten pro $\mathrm{mL}(\mathrm{IE} / \mathrm{mL})$ angegeben [27]. Die CEgekennzeichneten Tests variieren derzeit zum Teil relativ stark in einem weiten Bereich von 0,13-0,006 IE/mL. Der Stufenplan des PEI zur Bewertung von Tests im Spenderscreening [11] soll sicherstellen, dass nur sensitive HBsAg-Tests im Blutspenderscreening eingesetzt werden.

- Abb. 3b zeigt das Timing des HBVNachweises in der anti-HBc-negativen diagnostischen Fensterperiode einer akuten Hepatitis-B-Infektion. HBsAg wird durchschnittlich 37 Tage nach Infektion (bei einer infektiösen HBV-Dosis von $1 \mathrm{HBV}-\mathrm{DNA}-\mathrm{Kopie}$ ) nachgewiesen, gegenüber 32,1 bis 34,9 Tagen bei Minipool-(MP-)NAT, die überwiegend in Deutschland verwendet wird (LoD 89 bis 208 HBV-DNA-IE/mL bezogen auf die Einzelspende), und 19,2 Tagen bei Einzelspenden (ID-)NAT (LoD $\geq 2 \mathrm{IE} / \mathrm{mL}$; [28]). Die Bewertung des Zeitpunkts des 
Tab. 3 Charakterisierung von 188 Anti-HBc-reaktiven Proben von insgesamt 10.000 Spendern in zusätzlichen HBV-Markern und in 9 verschiedenen Anti-HBc-Tests

\begin{tabular}{|c|c|c|c|c|c|}
\hline \multirow[b]{3}{*}{ Gruppe } & \multirow[b]{3}{*}{ Reaktivität in zusätzlichen HBV-Markern } & \multirow[b]{3}{*}{ N Total (188) } & \multicolumn{3}{|c|}{ Anti-HBc-Reaktivität in 9 verschiedenen Anti-HBc-Tests } \\
\hline & & & \multicolumn{3}{|c|}{ Klasse } \\
\hline & & & A) 9 Tests & B) 5-8 Tests & C) $<5$ Tests \\
\hline 1) & Anti-HBc only & $13(6,9 \%)$ & 0 & 8 & 5 \\
\hline \multirow[t]{2}{*}{ 2) } & Anti-HBc + Anti-HBs & $62(33,5 \%)$ & 50 & 11 & 2 \\
\hline & Anti-HBc+ Anti-HBe & $7(3,7 \%)$ & 7 & 0 & 0 \\
\hline \multirow[t]{2}{*}{ 3) } & Anti-HBC + Anti-HBs + Anti-HBe & $105(55,9 \%)$ & 105 & 0 & 0 \\
\hline & Anti-HBC + Anti-HBs + HBV-DNA & $1(0,53 \%)$ & 1 & 0 & 0 \\
\hline- & - & - & $162(86,2 \%)$ & $19(10,1 \%)$ & $7(3,7 \%)$ \\
\hline \multirow[t]{2}{*}{-} & - & \multicolumn{4}{|c|}{ (168 (89,4 \%) insgesamt Anti-HBs positiv) } \\
\hline & & \multicolumn{4}{|c|}{ (112 (59,6\%) insgesamt Anti-HBe positiv) } \\
\hline \multicolumn{6}{|c|}{$\begin{array}{l}\text { Daten aus [35] } \\
\text { Gruppe nach Anzahl der detektierbaren HBV-Marker: 1) isoliert Anti-HBC reaktiv („Anti-HBC only"); 2) positiv auf einen zweiten HBV-Marker (Anti-HBs oder Anti- } \\
\text { HBe); 3) positiv auf } 3 \text { HBV-Marker (Anti-HBc plus Anti-HBs, plus Anti-HBe oder HBV-DNA) } \\
\text { Klasse nach Häufigkeit von Anti-HBc-positiv in allen } 9 \text { Anti-HBc-Tests: A) reaktiv in allen } 9 \text { Anti-HBC-Tests, B) reaktiv bei } 5-8 \text { Anti-HBC-Tests; C) reaktiv bei } 4 \text { oder } \\
\text { weniger Anti-HBc-Tests }\end{array}$} \\
\hline
\end{tabular}

HBV-Nachweises mit den verschiedenen HBV-Tests (HBsAg, MP- und IDNAT) ermöglicht einen Vergleich der Tests im Hinblick auf ihre Fähigkeit, das HBV-Übertragungsrisiko in der anti$\mathrm{HBc}$-negativen HBV-Fensterperiode $\mathrm{zu}$ verringern. Dieses war bei HBsAg mit $1: 1.270 .000$ bis $1: 1.620 .000$ bereits relativ gering und konnte durch MP-NAT (LoD $146 \mathrm{IE} / \mathrm{mL}$ ) um den Faktor 1,7 bzw. IDNAT um den Faktor 3,8 weiter reduziert werden. Es wird außerdem darauf hingewiesen, dass es neuere HBsAg-Tests gibt (Lumipulse G HBsAg-Quant, Architect HBsAg Next Qualitative, Alinity i HBsAg Next Qualitative; [29, 30]), die eine 4- bis 5-fach höhere Sensitivität aufweisen als die derzeit verwendeten HBsAg-Tests und damit in einem Sensitivitätsbereich liegen, der mit der Sensitivität von HBVDNA-Minipool-Testung vergleichbar ist [28].

Ein wichtiger weiterer Gesichtspunkt sind HBV-Genotypen und HBsAg-Mutanten, die die Sensitivität von HBsAgTests stark reduzieren können [28, 31-33]. In dieser Hinsicht erwies sich ein Testdesign als robust, bei dem mehrere Antikörper verwendet werden, um möglichst viele Epitope der HBsAg- ,a“Determinante abzudecken, z. B. 1 oder 2 monoklonale Antikörper auf der Festphase und mehrere monoklonale oder polyklonale Antikörper auf der Konjugatphase (Multiple/Poly- oder Mono/ Poly-Design; [33]), obwohl in seltenen
Fällen HBsAg-Mutanten weiterhin weniger gut nachgewiesen werden konnten [28].

\section{Nachweis des Hepatitis-B-Core- Antigens}

Während HBsAg und HBV-DNA nach der akuten Phase verschwinden können, bleiben Antikörper gegen das HepatitisB-Core-Antigen (Anti-HBc) in der Regel viele Jahre, oft lebenslang, erhalten. Anti$\mathrm{HBc}$ kann der einzige nachweisbare Marker einer abgeklungenen HBV-Infektion sein, wenn Anti-HBs auf nicht nachweisbare Werte zurückgeht und HBsAg sowie HBV-DNA nicht nachweisbar sind. Anti-HBc eignet sich daher zum Nachweis von potenziell infektiösen HBV-Spenden bei okkulter HBV-Infektion. Die derzeit verfügbaren CE-gekennzeichneten Anti$\mathrm{HBc}$-Tests sind relativ einheitlich hochsensitiv [34], haben eine Spezifität von mehr als 99,5\% bei Blutspendern [35] und liegen damit im gleichen Spezifitätsbereich wie bei anderen Screeningmarkern.

- Tab. 3 zeigt die Ergebnisse zu Anti$\mathrm{HBc}$ bei 10.000 Anti-HBc-vorgescreenten Blutspenden. Es gab 188 Anti-HBcreaktive Proben, die auf zusätzliche HBV-Marker und in 9 Anti-HBc-Tests detailliert analysiert wurden [35]: 89,4\% waren zusätzlich positiv für Anti-HBs, $59,6 \%$ positiv für Anti-HBe, alle waren HBsAg- und HBeAg-negativ, eine Pro- be war HBV-DNA-positiv, 6,9\% waren isoliert Anti-HBc-positiv („Anti-HBc only“; [36]); 86,2\% der 188 Anti-HBcpositiven Proben waren übereinstimmend in allen 9 Anti-HBc-Tests positiv, $10,1 \%$ in $\geq 5$ und $3,7 \%$ in $<5$ Anti-HBcTests. Diese Studien ergaben somit, dass die überwiegende Mehrheit der Anti$\mathrm{HBc}$-reaktiven Proben als echt positiv einzustufen war.

Nach Abklärung der Spezifität der Anti-HBc-Tests und weiterführenden Diskussionen, wonach von 1995 bis 2003 insgesamt 18 gesicherte HBV-Übertragungen ans PEI gemeldet wurden, wovon 7 durch Anti-HBc-Testung zu verhindern gewesen wären, folgte ein Beschluss des Arbeitskreises Blut [37]. Dies führte 2006 schließlich zur Einführung der Spendertestung auf Antikörper gegen HepatitisB-Core-Antigen [10] und zum aktuellen Testalgorithmus des Arbeitskreises Blut [38] für die Bestätigung des HBV-Status durch eine hochsensitive HBV-DNANAT $(\leq 5 \mathrm{IE} / \mathrm{mL})$ oder mindestens einen zusätzlichen Anti-HBc-Test in einem anderen Testformat. Dieser Anti-HBc-Abklärungsalgorithmus wurde in Blutspendeeinrichtungen im Wesentlichen bestätigt [39] mit nur geringfügigen Unterschieden und ohne, dass dabei die Virussicherheit beeinträchtigt war. 


\section{Erfolg der regulatorischen Maßnahmen}

Sowohl jeder begründete Verdacht als auch der Nachweis einer transfusionsbedingen Infektion sind meldepflichtig (gemäß $\$ 19$ Abs. 1 TFG) und unverzüglich dem PEI anzuzeigen. Dieses etablierte Meldesystem trägt dazu bei, den Erfolg eingeführter Maßnahmen und somit die Sicherheit von in Deutschland verwendeten Blutprodukten systematisch und stetig zu bewerten.

Die verpflichtende Einführung der HCV-NAT-Testung von Blutspenden im Jahr 1999 zeigte einen direkten und deutlichen Erfolg für die Sicherheit von Blut und Blutprodukten. Während von 1997 bis 1998 noch insgesamt 19 transfusionsbedingte HCV-Infektionen registriert wurden, beschränkt sich die Anzahl der berichteten Übertragungen in den folgenden 20 Jahren (von 1999 bis 2019) auf 3 nachgewiesene Fälle [40-42]. Die 3 Übertragungsfälle gehen auf 2 Blutspenden während der frühen diagnostischen Fensterperiode der Infektion zurück, wobei die HCV-Konzentrationen im Blut zu gering waren, um sie mit der verwendeten NAT-Methode zu detektieren [43, 44]. Zusätzlich wurden im Zeitraum von 1999 bis 2015 in Deutschland 153 Blutproben als HCVpositiv nachgewiesen, die ohne HCVNAT nicht identifiziert worden wären $[40,41]$, was wiederum die Wichtigkeit der HCV-NAT-Testung unterstreicht.

Die Einführung der Anti-HBc-Testung im Jahr 2006 zusammen mit der freiwilligen Etablierung der HBV-NAT zur etwa gleichen Zeit in den meisten Blutspendeeinrichtungen hatte einen ähnlich positiven Effekt auf die Anzahl der Virusübertragungen wie oben für HCV beschrieben. Von 1997 bis 2006 wurden durchschnittlich 2 transfusionsbedingte HBV-Infektionen pro Jahr in Deutschland nachgewiesen [41]. Seit 2007 wurde die Zahl auf insgesamt noch 5 HBV-Übertragungsfälle reduziert (Daten verfügbar bis einschließlich 2019; [28, 42, 45]). Aufgrund der simultanen Einführung der Anti-HBcTestung zusammen mit der HBV-NATTestung in mittlerweile fast allen deutschen Blutspendeeinrichtungen kann der Rückgang der transfusionsbedingen HBV-Übertragungsfälle nicht eindeutig auf eine der beiden Nachweismethoden zurückgeführt werden. Unter den 2019 im Rückverfolgungsverfahren bestätigten HBV-positiven Blutspenden befanden sich Fälle, die im initialen Screening isoliert positiv für jeweils nur einen der 3 Marker (HBsAg, Anti-HBc, HBVNAT) getestet wurden [42]. Angesichts der geringen HBV-Übertragungsfälle der letzten Jahre und somit einer hohen Sicherheitslage besteht aktuell kein definierbar hohes Risiko, welches eine Änderung des gültigen Testalgorithmus bedingt.

Ob die Verpflichtung zur HEV-NAT seit 2020 einen vergleichbaren Erfolg für die Sicherheit von Blutprodukten erzielt, wird sich in den nächsten Jahren zeigen. Eine Modellierung von 2017 basierend auf Daten zur HEV-Prävalenz bei Blutspendern in Deutschland zeigte auf, dass die Einführung einer HEVNAT mit einer angenommenen PoolgröBe von 96 und Sensitivität von $20 \mathrm{IE} / \mathrm{mL}$ $80 \%$ der erwarteten HEV-Übertragungen und die daraus resultierenden chronischen Infektionen verhindern würde [46]. Seit 2013, dem Beginn der Aufzeichnung von transfusionsbedingten HEVInfektionen, wurden dem PEI bis einschließlich 201923 HEV-Übertragungen gemeldet - 10 davon allein 2019 [42]. Die Dunkelziffer kann hier aber durchaus noch höher liegen, da die Aufmerksamkeit für HEV-Infektionen erst in den letzten Jahren stetig angestiegen ist. Im Jahr 2020, dem ersten Jahr mit verpflichtender HEV-Testung, konnte bereits ein erster Effekt der Maßnahme auf die PEI-Meldedaten beobachtet werden. So hat sich die Anzahl der eingeleiteten Rückverfolgungsverfahren für HEV von $947 \mathrm{im} \mathrm{Jahr}$ 2019 auf 2771 im Jahr 2020 fast verdreifacht. Gleichzeitig wurde 2020 nur eine transfusionsbedingte HEV-Übertragung gemeldet [47]. Ob dieser Trend auch in den nächsten Jahren anhält, wird weiter beobachtet werden.

\section{Fazit}

Seit den vermehrten Virusübertragungsfällen der 1980er- und 1990er-Jahre konnte die Sicherheit von Blut und
Blutprodukten durch Einführung neuer Testanforderungen und deren kontinuierliche Anpassung fortlaufend verbessert und dem Stand der Technik angepasst werden. Mithilfe der etablierten serologischen und NAT-basierten Nachweismethoden bei Blutspenden konnte somit die Anzahl an transfusionsbedingten HCV- und HBV-Infektionen in den letzten 20 Jahren auf ein Minimum mit wenigen Fällen reduziert werden. Mit Einführung der HEV-Spendertestung im Jahr 2020 ist die Wahrscheinlichkeit hoch, auch die gemeldeten HEVÜbertragungen auf seltene Einzelfälle zu minimieren. Aufgrund der zugrunde liegenden Regulationsstruktur ist es auch in Zukunft möglich, die Testalgorithmen bei Bedarf an neue Datenlagen anzupassen, um die Sicherheit von Blutspenden weiterhin zu gewährleisten. Bei jährlich etwa 4 Mio. Blutspenden in Deutschland kann die Sicherheit von Blutprodukten insgesamt als sehr hoch und das Risiko einer Übertragung mit Hepatitisviren als sehr gering bewertet werden.

\section{Korrespondenzadresse}

\section{Dr. Julia Kreß}

Fachgebiet Molekulare Virologie, Paul-EhrlichInstitut

Paul-Ehrlich-Straße 51-59, 63225 Langen,

Deutschland

Julia.Kress@pei.de

Danksagung. Wir danken Olaf Henseler für die Zusammenstellung der IVD-Daten.

Funding. Open Access funding enabled and organized by Projekt DEAL.

\section{Einhaltung ethischer Richtlinien}

Interessenkonflikt. J. Mitterreiter, H. Scheiblauer, S. Fiedler und J. Kreß geben an, dass kein Interessenkonflikt besteht.

Für diesen Beitrag wurden von den Autoren keine Studien an Menschen oder Tieren durchgeführt. Für die aufgeführten Studien gelten die jeweils dort angegebenen ethischen Richtlinien.

Open Access. Dieser Artikel wird unter der Creative Commons Namensnennung 4.0 International Lizenz veröffentlicht, welche die Nutzung, Vervielfältigung, Bearbeitung, Verbreitung und Wiedergabe in jeglichem Medium und Format erlaubt, sofern Sie den/die ursprünglichen Autor(en) und die Quelle ordnungsgemäß nennen, einen Link zur Creative Commons Lizenz 
beifügen und angeben, ob Änderungen vorgenommen wurden.

Die in diesem Artikel enthaltenen Bilder und sonstiges Drittmaterial unterliegen ebenfalls der genannten Creative Commons Lizenz, sofern sich aus der Abbildungslegende nichts anderes ergibt. Sofern das betreffende Material nicht unter der genannten Creative Commons Lizenz steht und die betreffende Handlung nicht nach gesetzlichen Vorschriften erlaubt ist, ist für die oben aufgeführten Weiterverwendungen des Materials die Einwilligung des jeweiligen Rechteinhabers einzuholen.

Weitere Details zur Lizenz entnehmen Sie bitte der Lizenzinformation auf http://creativecommons.org/ licenses/by/4.0/deed.de.

\section{Literatur}

1. World Health Organization (2017) Global hepatitis report 2017. World Health Organization, Geneva (Licence: CCBY-NC-SA3.0IGO)

2. Robert Koch-Institut (2020) Infektionsepidemiologisches Jahrbuch meldepflichtiger Krankheiten für 2019 https://doi.org/10.25646/6948

3. Robert Koch-Institut (2015) Epidemiologisches Bulletin Nr. 15 https://doi.org/10.25646/4706

4. Hewitt PE, ljaz S, Brailsford SR et al (2014) Hepatitis E virus in blood components: a prevalence and transmission study in southeast England. Lancet 384:1766-1773. https://doi.org/10.1016/S01406736(14)61034-5

5. Official Journal of the European Union: Directive 2002/98/EC of the European Parliament and of the Council of 27 January 2003 setting standards of quality and safety for the collection, testing, processing, storage and distribution of human blood and blood components and amending Directive 2001/83/EC. 2003

6. Transfusionsgesetz. http://www.gesetze-iminternet.de/tfg/index.html. Zugegriffen: 2. Aug. 2021

7. Arzneimittelgesetz. https://www.gesetze-iminternet.de/amg_1976/.Zugegriffen:2.Aug. 2021

8. Bekanntmachung der Richtlinie zur Gewinnung von Blut und Blutbestandteilen und zur Anwendung von Blutprodukten (Richtlinie Hämotherapie) gemäß den $\S \S 12 a$ und 18 des Transfusionsgesetzes - vom 4. Oktober 2017. Bundesanzeiger BAnz AT 6. Nov. 2017

9. Bekanntmachung über die Ergebnisse des Stufenplanverfahrens zur Verminderung des Risikos von Hepatitis B-, Hepatitis C- und HIV-Infektionen bei Empfängern von Erythrozytenkonzentraten (BAnz.Nr.63vom 04.04.97,S.4477) vom25.Februar 1998. Bundesanzeiger 1998;53: 12269-70

10. Abwehr von Arzneimittelrisiken; Testung auf Antikörper gegen Hepatitis-B-Core-Antigen (anti$\mathrm{HBC}$ ) im Blutspendewesen (vom 08. Mai 2006). Bundesanzeiger Nr. 109 vom 13. Juni 2006, S. 4370 (neugefasst durch Bekanntmachung im Bundesanzeiger BAnz AT 18. März 2014 B6)

11. Bekanntmachung - Abwehr von Arzneimittelrisiken - Anordnung von Auflagen nach $\S 28$ zu den Zulassungen für zelluläre Blutkomponente und gefrorene Frischplasmen - Vom 7. Januar 2013. Bundesanzeiger BAnz AT 19. Febr. 2013B

12. Bekanntmachung über die Zulassung von Arzneimitteln - Abwehr von Arzneimittelrisiken Anordnung der Testung von Blutspendern zur Verhinderung einer Übertragung von HepatitisE-Virus durch Blutkomponenten zur Transfusion und Stammzellzubereitungen zur hämatopoetischen Rekonstitution - vom 5. Februar 2019. BundesanzeigerBAnz AT 17. Mai 2019B7

13. Robert Koch-Institut (2020) Epidemiologisches Bulletin 30/31 https://doi.org/10.25646/7032

14. Arbeitskreis Blut (2000) Hepatitis-B-Virus (HBV). Stellungnahmen des Arbeitskreises Blut. Bundesgesundheitsblatt Gesundheitsforschung Gesundheitsschutz 43:240-248

15. Shin E-C, Sung PS, Park S-H (2016) Immune responses and immunopathology in acute and chronic viral hepatitis. Nat Rev Immunol 16:509-523. https://doi.org/10.1038/nri.2016.69

16. Arbeitskreis Blut (2003) Hepatitis-C-Virus (HCV). Stellungnahmen des Arbeitskreises Blut. Bundesgesundheitsblatt Gesundheitsforschung Gesundheitsschutz 46:712-722

17. Page-Shafer K, Pappalardo BL, Tobler LH et al (2008) Testing strategy to identify cases of acute hepatitis Cvirus (HCV) infection and to project HCV incidence rates. J Clin Microbiol 46(2):499-506. https://doi.org/10.1128/JCM.01229-07

18. Juhl D, Baylis SA, Blümel J, Görg S, Hennig H (2014) Seroprevalence and incidence of hepatitis $E$ virus infection in German blood donors. Transfusion 54:49-56

19. Vollmer $T$, Diekmann J, Johne R, Eberhardt $M$, Knabbe C, Dreier J (2012) Novel approach for detection of hepatitis $E$ virus infection in German blood donors. J Clin Microbiol 50:2708-2713. https://doi.org/10.1128/JCM.01119-12

20. Westhölter D, Hiller J, Denzer U et al (2018) HEVpositive blood donations represent a relevant infection risk for immunosuppressed recipients. J Hepatol 69:36-42. https://doi.org/10.1016/j. jhep.2018.02.031

21. Kamar N, Bendall R, Legrand-Abravanel $F$ et al (2012) Hepatitis E. Lancet 379:2477-2488. https:// doi.org/10.1016/S0140-6736(11)61849-7

22. Mackay IM, Arden KE, Nitsche A (2002) Real-time PCR in virology. Nucleic Acids Res 30:1292-1305. https://doi.org/10.1093/nar/30.6.1292

23. Hill CS (2001) Molecular diagnostic testing for infectious diseases using TMA technology. Expert Rev Mol Diagn 1:445-455. https://doi.org/10. 1586/14737159.1.4.445

24. Roth WK, Weber M, Seifried E (1999) Feasibility and efficacy of routine PCR screening of blood donations for hepatitis $C$ virus, hepatitis $B$ virus, and HIV-1 in a blood-bank setting. Lancet 353(9150):359-363. https://doi.org/10.1016/ S0140-6736(98)06318-1

25. Nick S, Scheiblauer H (2007) Sensitivities of CEmarked HIV, HCV, and HBsAg assays. J Med Virol 79:S59-S64. https://doi.org/10.1002/jmv.20965

26. Scheiblauer H, El-Nageh M, NickS, Fields H,Prince A, Diaz S (2006) Evaluation of the performance of 44 assays used in countries with limited resources for the detection of antibodies to hepatitis $C$ virus. Transfusion 46:708-718. https://doi.org/10.1111/ j.1537-2995.2006.00789.x

27. Wilkinson DE, Seiz PL, Schuttler CG et al (2016) International collaborative study on the 3rd WHO International Standard for hepatitis B surface antigen. J Clin Virol 82:173-180. https://doi.org/ 10.1016/j.jcv.2016.06.003

28. Scheiblauer $H$, Heiden $M$, Funk $M$ et al (2020) Detection of hepatitis B virus infection in German blood donors 2008-2015. Vox Sang 115:152-161. https://doi.org/10.1111/vox.12890

29. Yang R, Song G, Guan W, Wang Q, Liu Y, Wei L (2016) The Lumipulse G HBsAg-Quant assay for screening and quantification of the hepatitis $B$ surface antigen. J Virol Methods 228:39-47. https://doi org/10.1016/j.jviromet.2015.11.016

30. Lou S, Taylor R, Pearce S, Kuhns M, Leary T (2018) An ultra-sensitive Abbott ARCHITECT ${ }^{\circledR}$ assay for the detection of hepatitis $B$ virus surface antigen (HBsAg). J Clin Virol 105:18-25. https://doi.org/10. 1016/j.jcv.2018.05.009

31. Chudy M, Scheiblauer $H$, Hanschmann K-M et al (2013) Performance of hepatitis B surface antigen tests with the first WHO international hepatitis $B$ virus genotype reference panel. J Clin Virol 58:47-53. https://doi.org/10.1016/j.jcv.2013.06. 011

32. Scheiblauer H, Soboll H, Nick S (2006) Evaluation of 17 CE-Marked HBsAg Assays With Respect to Clinical Sensitivity, Analytical Sensitivity, and Hepatitis B Virus Mutant Detection. J Med Virol 78(Suppl 1):S66-S70. https://doi.org/10.1002/ jmv.20611

33. Scheiblauer H, El-Nageh M, Diaz S et al (2010) Performance evaluation of 70 hepatitis $B$ virus (HBV) surface antigen (HBsAg) assays from around the world by a geographically diverse panel with an array of HBV genotypes and HBsAg subtypes. Vox Sang 98:403-414. https://doi.org/10.1111/j. 1423-0410.2009.01272.x

34. Scheiblauer H, Ferguson M, Volkers P, NickS, World Health Organization, Biologicals Unit, WHO Expert Committee on Biological Standardization (2008) Report of the WHO collaborative study to establish the first international standard for detection of antibodies to hepatitis B core antigen (anti-HBc), human plasma. https://apps.who.int/iris/handle/ 10665/69975.Zugegriffen: 1.Aug. 2021

35. Schmidt M, Nubling CM, Scheiblauer Het al (2006) Anti-HBc screening of blood donors: a comparison of nine anti-HBc tests. Vox Sang 91:237-243. https://doi.org/10.1111/j.1423-0410.2006.00818.

36. GrobP,JilgW,BornhakHetal (2000) Serological pattern "anti-HBcalone": Report on a workshop. J Med Virol 62:450-455. https://doi.org/10.1002/10969071(200012)62:4/450:AID-JMV9)3.0.CO;2-Y

37. Arbeitskreis Blut (2005) Erhöhung der Sicherheit von zellulären Blutkomponenten und quarantänegelagertem Frischplasma durch Untersuchung der Blut- und Plasmaspenden auf Antikörper gegen das Hepatitis-B-Core Antigen (Anti-HBC). Bundesgesundheitsblatt 48:698-699. https://doi. org/10.1007/s00103-005-1067-9

38. Arbeitskreis Blut (2020) Verfahren zur Rückverfolgung (Look Back) gemäß § 19 Transfusionsgesetz. Bundesgesundheitsblatt 64:108-125. https://doi. org/10.1007/s00103-020-03258-w

39. Juhl D, Knobloch JK, Görg S, Hennig H (2016) Comparison of two test strategies for clarification of reactive results for anti-HBc in blood donors. Transfus Med Hemother 43(1):37-43. https://doi. org/10.1159/000441676

40. Fiedler SA, Oberle D, Chudy M et al (2019) Effectiveness of blood donor screening by HIV HCV, HBV-NAT assays, as well as HBsAg and antiHBc immunoassays in Germany (2008-2015). Vox Sang 114:443-450. https://doi.org/10.1111/vox. 12770

41. Nübling CM, Heiden M, Chudy $M$ et al (2009) Experience of mandatory nucleic acid test (NAT) screening across all blood organizations in Germany: NAT yield versus breakthrough transmissions. Transfusion 49:1850-1858. https:// doi.org/10.1111/j.1537-2995.2009.02212.x

42. Funk MB, Heiden M, Müller S et al (2021) Hämovigilanz-Bericht des Paul-Ehrlich-Instituts 
2019: Auswertung der Meldungen von Reaktionen und Zwischenfällen nach §63i AMG

43. Kretzschmar E, Chudy M, Nübling CM, Ross RS Kruse F, Trobisch H (2007) First case of hepatitis C virus transmission by a red blood cell concentrate after introduction of nucleic acid amplification technique screening in Germany: a comparative study with various assays. Vox Sang 0:297-301. https://doi.org/10.1111/j.1423-0410.2007.00903. $x$

44. Himmelsbach K, Müller S, Reissinger A et al (2021) Second Hepatitis $C$ virus transmission by blood components since mandatory NAT screening in Germany. Transfusion (in review)

45. Funk MB, Heiden M, Müller S et al (2020) Hämovigilanz-Bericht des Paul-Ehrlich-Instituts 2018: Auswertung der Meldungen von Reaktionen und Zwischenfällen nach $\S 63 \mathrm{i} A M G$

46. Kamp C, Blümel J, Baylis SA et al (2018) Impact of hepatitis $E$ virus testing on the safety of blood components in Germany — results of a simulation study. Vox Sang 113:811-813. https://doi.org/10. 1111/vox.12719

47. Hämovigilanz-Berichte des Paul-Ehrlich-Institutes. https://www.pei.de/haemovigilanzbericht. Zugegriffen:23.Sept. 2021 